\title{
Atomic Force Microscopy to explore Electroporation effects on cells
}

\author{
Etienne Dague \\ LAAS-CNRS, Université de Toulouse, CNRS, Toulouse France \\ edague@laas.fr
}

\begin{abstract}
Atomic Force Microscopy (AFM) is more and more used in life science. Its ability to provide images of living cells as well as mechanical or adhesion maps makes it a technology that cannot be ignored. In the context of electroporation (EP) which undoubtly affects the cells membrane or wall, a technology able to probe the cell surface is more than interesting. This chapter describes the principle of the AFM technology and especially the latest multiparametric imaging modes developed recently. It then demonstrates that AFM can be used to probe cell's morphology modifications induced by electric pulses. We then show that EP modifies cell's nanomechanical properties and that the actin cytoskeleton plays a major role in this process. Finally we shed light on the effects of EP on bacteria as probed by AFM. In this latest example it must be noticed that no mechanical modifications are induced, but the adhesion properties of the bacteria are dramatically reduced by Pulsed Eelectric Fields (PEF). Altogether the chapter shows the interest of applying AFM on cells exposed to EP, in order to get a better fundamental understanding of EP effect on cells or bacteria.
\end{abstract}

Keywords: Atomic Force Microscopy, Electroporation, Elasticity, Cell topography, cytoskeleton, bacteria, adhesion 


\section{Introduction}

Electroporation (EP) consists in applying electric fields of various intensity and/or duration on cells in order to modify their interface and let non-permeant molecules go through the plasmic membrane $^{1}$. EP has proven to be efficient to potentiate cytotoxic molecules (electrochemotherapy in cancer treatment) and has the potential to be used in gene transfer ${ }^{2,3}$. However the mechanisms of cell membrane modifications are poorly understood and limit the applications of this technology ${ }^{4}$. Exploring and understanding the modifications induced by EP on cell's membrane is indeed a challenge that has been addressed by measuring the entry of fluorescent or radioactive molecules in pulsed cells or by transmembrane potential measurements. However using these techniques, the direct physical effect on the membrane is not obvious and the word electroporation itself is questionable as no pores were ever reported. Electropermeabilization is therefore sometimes preferred. The cell membrane permeability is, indeed, modified and understanding electroporation effects is therefore a matter of cell membrane exploration. In this context the atomic force microscope is a pertinent technology as it makes it possible to analyze cells surface in their native environment, in this case before and after electric pulses application. Atomic Force Microscopy (AFM) is an imaging technology developed in the $80^{\text {th }}$ by physicists ${ }^{5-7}$. It has been more and more applied on biological samples (virus, bacteria, mammalian cells, DNA, proteins...) since the $90^{\text {th } 8}$ and recently to explore the effects of EP on cells morphology and nanomechanical properties ${ }^{9-13}$. But more than an imaging technology AFM is also a force machine able to measure forces in the $\mathrm{pN}$ range $\mathrm{e}^{14}$. This capability can be used to measure the nanomechanical properties of cells and especially after EP.

In this chapter I will describe the AFM technology and present its application to better understand EP. 


\section{Atomic Force microscopy}

The AFM principle ${ }^{5}$ relies on the measure of interaction forces between a sharp tip and the analyzed sample. The tip is scanned over the sample, or conversely, the sample is scanned over the tip, thanks to a piezo electric ceramic that can be moved in the 3 spatial directions: $\mathrm{x}$, $\mathrm{y}$ and $\mathrm{z}$. In order to allow a displacement of the tip in the $\mathrm{z}$ direction, it is mounted on a cantilever, which deflection is monitored via an optical device based on a laser and a 4 quadrants photodiode. The resolution is therefore directly linked to the tip size. The sharpest tip, like for example carbon nanotubes, will achieve the highest resolution. The drawback is that such tips are both stiff and delicate. As a consequence they are not adapted for living cells and tips presenting pyramidal geometry (curvature radius around $20 \mathrm{~nm}$ ) are preferred for living cells exploration. Another parameter that can limit AFM resolution is the scanning velocity. Images, or force maps are recorded line-by-line, or point-by-point and this is a time consuming process. A 512 lines image recorded at a scan rate of $1 \mathrm{~Hz}$ requires 8 minutes and 30 seconds (each line is scanned 2 times). A solution to increase the resolution can be to decrease the scanned area. Classical piezo can scan areas up to $100 \times 100 \mu \mathrm{m}^{2}$. By decreasing the analyzed surface, the resolution is mechanically increased. However this has a limit linked to the tip size. In a $10 \times 10 \mu \mathrm{m}^{2}$ picture made of 512 lines, the thickness of each line is of 19 $\mathrm{nm}$, what is close to the typical curvature radius of tips used for living cells. In this case, decreasing the scan size will not result in increasing the resolution so much, each line being smaller than the tip size.

The main advantage of AFM in life science is its ability to work in liquids, like growing media; at a controlled temperature, i.e. $37^{\circ} \mathrm{C}$; under gas flow, typically $\mathrm{CO}_{2} 5 \%$. In these conditions living cells can be observed during several hours, in their native condition and/or after a treatment (drug, EP, temperature modification...). 


\section{Imaging}

AFM images represent the topography of the sample, which is deduced from the cantilever deflection. In contact mode (figure 1), the tip is applying a constant and arbitrary force on the sample during scanning. The force is kept constant by modulating the tip or sample height trough a feedback loop. This mode is easy to use and provides high quality images. However the tip, while scanning, induces lateral forces that can damage the sample, especially in the case of a soft sample like a cell. Conversely the lateral forces induce a friction, which is recorded as a lateral deflection of the tip. This lateral deflection is material dependent and can therefore be used to decipher between materials under investigation. The drawback of these lateral forces is that the biological sample has to be firmly immobilized on the surface to overcome them (figure 1). Thus strategies were developed to perform non denaturing immobilization ${ }^{15-18}$. It must be noticed that mammalian cells are usually spreading on their support and that no special immobilization procedure is needed (figure 1). However, these cells are very soft (Young modulus (YM) are ranging from 1 to $100 \mathrm{kPa}$ ) and are usually difficult to image in contact mode.

To overcome this limitation the historical AFM manufacturer developed a non-contact mode named Tapping ${ }^{\circledR}$ mode. Here the tip is oscillated near to its resonance frequency, and the oscillation amplitude is kept constant during scanning. The phase shift can also be interpreted as material dependent, and the phase signal used to decipher between different materials. 


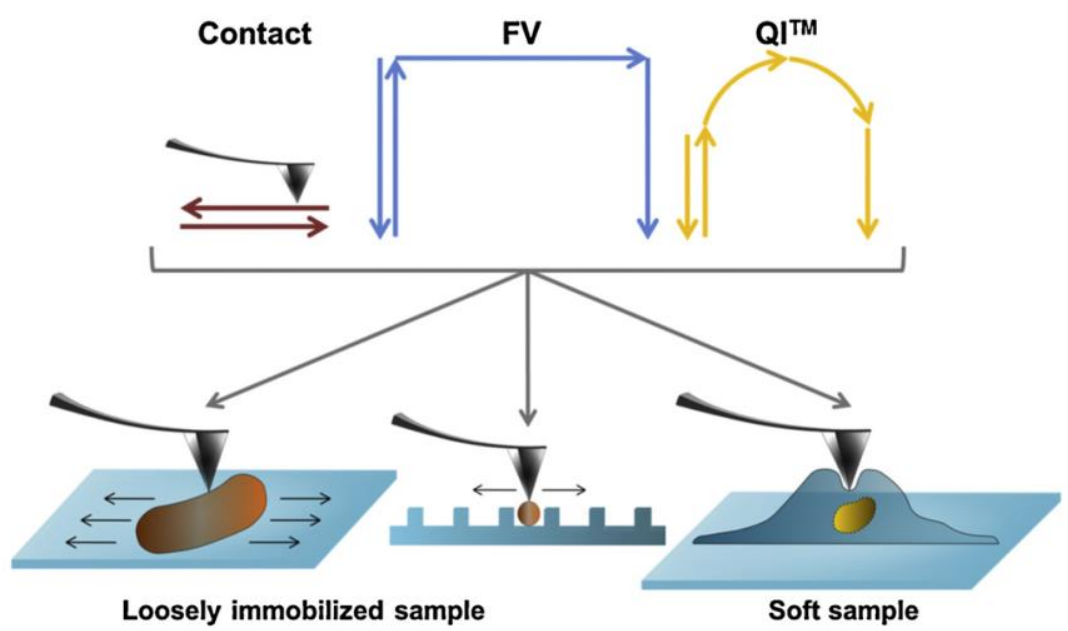

Figure 1: Schematic representation of 3 AFM modes: contact, Force Volume (FV) and Quantitative imaging. These modes can be used to explore loosely immobilized sample, samples trap in holes of a PolyDi-Methyl-Siloxane (PDMS) stamp or soft samples such as mammalian cells (adapted from ${ }^{10}$ ).

\section{Force Spectroscopy: nanomechanics, adhesion.}

As already stated, the AFM is a force machine. In the force spectroscopy mode, the tip is no longer scanned in the $\mathrm{x}, \mathrm{y}$ direction but in the $\mathrm{z}$ direction. While approaching the sample, the tip is first encountering no forces, then it contacts the surface and finally the cantilever and the sample (if it is soft) are deflected. The approach force curve can be converted into an indentation force curve. From the indentation of the tip in the sample, the mechanical properties can be deduced, using models like the Hertz model. In this model, as in many others, the applied force is a function of the sample elasticity or Young Modulus. A higher value of $\mathrm{E}$ means that a higher force is required to achieve the same indentation in the sample. Thus the link between elasticity and the Young modulus is biologically unintuitive: high Young modulus do not mean easily deformable. The Young modulus is a pressure measured in $\mathrm{Pa}\left(\mathrm{N} \cdot \mathrm{m}^{-2}\right)$. The exact absolute value of a sample Young modulus is a complex question. The sample anisotropy, the shape of the tip sample contact, the dispersed energy, the sample viscosity, etc... should be taken into account to achieve an exact measure. For this reason, Young modulus absolute values should be used with caution. Nevertheless, the comparison of Young modulus values measured on the same sample, but in different conditions is valuable 
and provides interesting insight into the nanomechanical modifications induced by a treatment.

During the retraction process, the cantilever comes back to zero deflection, but if an interaction has occurred between the tip and the sample, a higher force will be needed to detach the tip from the sample. The adhesion forces can be due to electrostatic or hydrophobic interactions $^{19,20}$ or to specific biological interactions between a ligand and a receptor for example $^{21,22}$. Measuring specific forces require to functionalize the tip, which can be performed through the use of linkers grafted on the AFM tip.

In the Force Volume (FV) mode (Figure 1), force curves are recorded according to a matrix of point. Each force curve is recorded and their analysis leads to adhesion and/or elasticity maps.

\section{Multiparametric modes}

Multiparametric modes ${ }^{23}$ (figure 1) were developed recently by two AFM manufacturers. They are optimizing the FV by increasing the number of force curves recorded in the same time. The resolution is therefore increased and/or the acquisition time is reduced. In this new mode, and thanks to an increased resolution, both, valuable topography, adhesion and elasticity maps are recorded at the same time. Moreover, multiparametric modes are convenient to image soft and loosely immobilized biological samples. Indeed, as the tip is retracted between each force curves, thus no or low lateral forces are exerted on the sample. The main limitation remaining in these modes is the $\mathrm{z}$ piezo course. It must be large enough to detach the tip from the cell; however this inconvenient can be avoided by a selecting the appropriate tip (shape) and cantilever (spring constant)

\section{Imaging effects of EP on cells}


In order to explore EP effects on mammalian cells shape, Chopinet et al. ${ }^{9}$, in 2013, first imaged, by AFM, cells immediately fixed after EP. No specific morphological alterations were detected neither in front of the electrode nor on regions perpendicular to the electrodes. Globally the measured elasticity is decreased after EP (from $30.0+/-2.0 \mathrm{kPa}$ to $20.5+/-1.0$ $\mathrm{kPa}$ ), but no link with the electrodes position appears. Then living cells were imaged across time. Figure $2 \mathrm{~A}$ presents the same living cell imaged during 50 minutes and figure $2 \mathrm{C}$ cross sections of the cell measured after 9,24 and 50 minutes of imaging. These topographical images were recorded in the multiparametric quantitative imaging mode. It demonstrates that the cells are not changing their shape during a 50 minutes period of time. At higher resolution (see insets in the down right corners and supplementary material in ${ }^{9}$ ), images show that the membrane is smooth, homogeneous and cytoskeleton fibers are detected. The membrane and cytoskeleton remain stable during all the experiment.
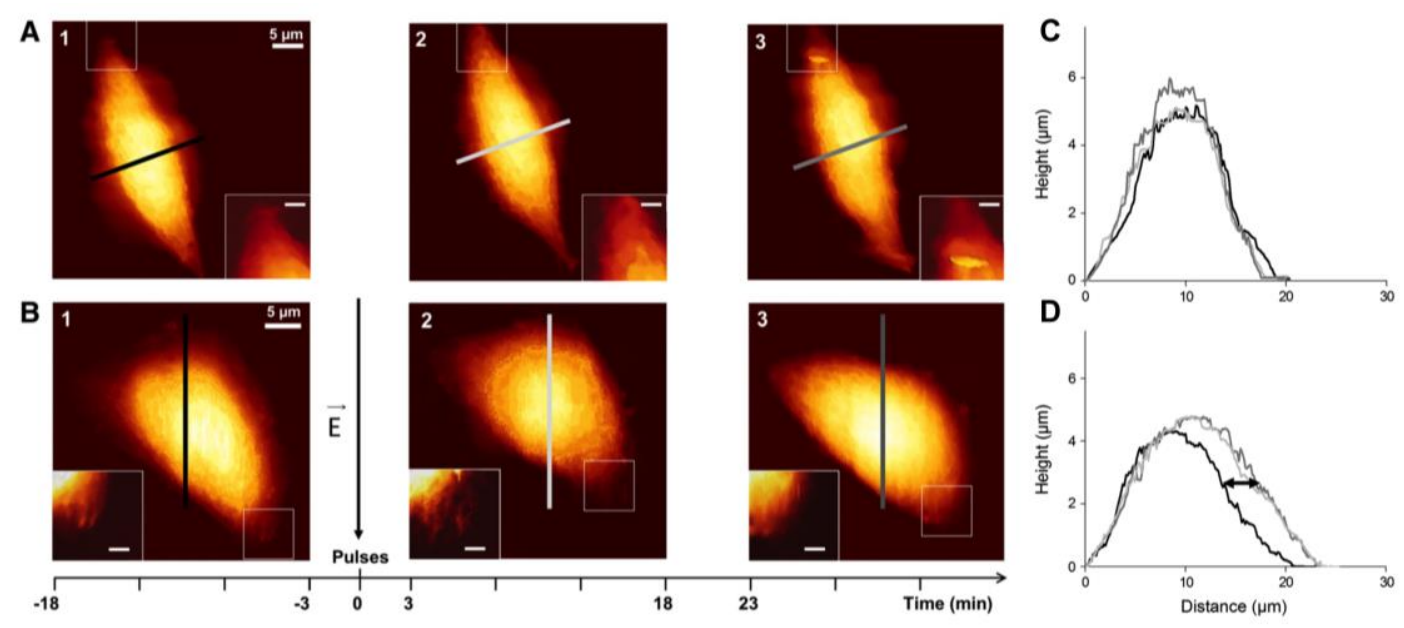

Figure 2: AFM QI images of living cells before and after EP. A 1-3: 3 AFM height images of the same untreated cell across time. B 1: AFM height image of an untreated cell; B 2-3: AFM height images of the cell presented in $B 1$ respectively 18 and 50 minutes after EP. The scale bar in the insets represents $1 \mu \mathrm{m}$. The insets present details of the membrane and are numerical zoom. $C$, D: cross sections of the cells taken along the lines indicated for the control cell (C) and the pulsed cell (D); the black line corresponds to non pulsed cells, light grey 15 minutes later and dark-grey 30 minutes later. The double arrow in D marks the cell swelling. The color scale is ranging from 0 to $7 \mu \mathrm{m}$. Adapted from ${ }^{9}$

In a second step, figure $2 \mathrm{~B}, \mathrm{EP}$ ( 8 square-wave electric pulses of $5 \mathrm{~ms}$ duration at $400 \mathrm{~V} / \mathrm{cm}$ )

was applied at a frequency of $1 \mathrm{~Hz}$ through stainless steel parallel electrodes directly on the

Petri dish in buffer after the first image of the cell. The next 2 images and the cross sections 
presented figure 2D (black arrow) demonstrate a swelling of the cell induced by EP. At higher resolution, cell membrane modifications appear to be reversible. After 18 minutes: i) many extensions rippling membrane can be seen and ii) no cytoskeleton fibers are observed any more. At the end of the experiment (50 minutes) the cell membrane seems to recover, looking like before EP.

EP is also used in food industry to eradicate undesirable bacteria. Pillet et al. ${ }^{12}$, in 2016 explored the effect of EP ( $5 \mu$ s-pulses at frequency of $1 \mathrm{kHz}$ in a $4.1 \mathrm{mM} \mathrm{NaCl}$ solution, with a conductivity of $500 \mu \mathrm{S} / \mathrm{cm}$ and a $\mathrm{pH}$ of 7), on Bacillus pumilus cell surface and shape. They both analyzed vegetative bacteria and spores. Figure 3 a, b, c and d presents AFM height images of vegetative cells before $(a, c)$ and after EP $(b, d)$.
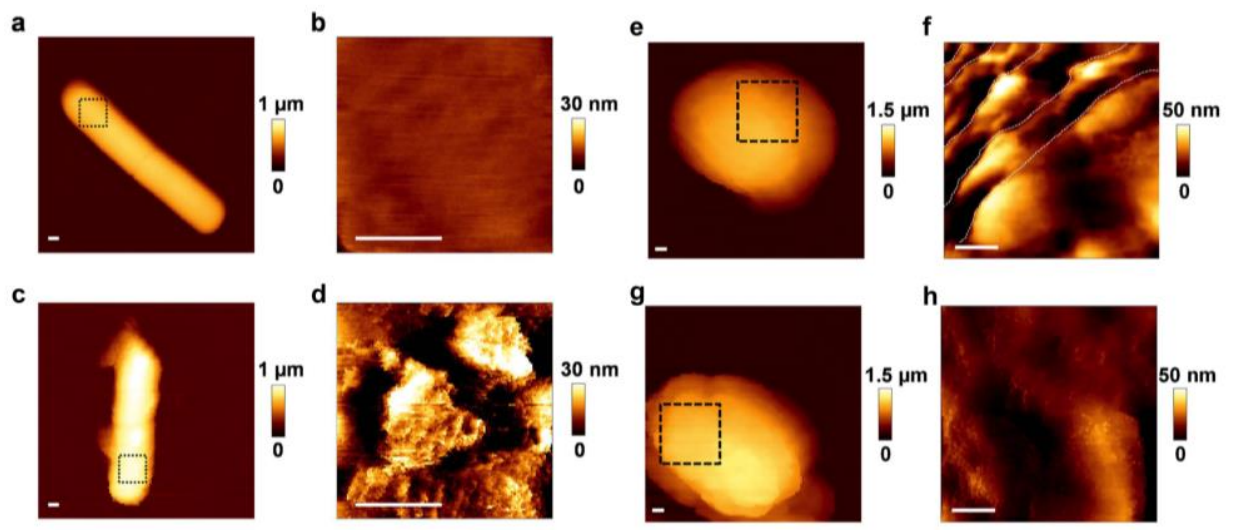

Figure 3: AFM QI images of Bacillus pumilus vegetative cells (a, b, c and d; scale bars: $200 \mathrm{~nm})$ and spores (e, $f, g$ and $h$; scale bars: $100 \mathrm{~nm})$ before $(a, b, e$ and $f)$ and after (c, $d, g$ and $h)$ EP. The dotted scares indicate the position of images $b, d, f$, and $h$ respectively on images a, $c, e$ and $g$. The color scale, representing the highness, is given on the right of each image. Adapted from ${ }^{12}$

The surface roughness has been determined to be $1.4+/-0.5 \mathrm{~nm}$ before EP and $6.1+/-5.2$

after EP. Similarly to mammalian cells, EP induces a swelling of vegetative bacteria: the mean cell volume is $1.9+/-0.7 \mu \mathrm{m}^{3}$ before EP and increases to $3.0+/-1.6 \mu \mathrm{m}^{3}$ after pulses application. Vegetative bacteria have thus a smooth surface that is dramatically impaired by EP. The effect is emphasized on higher resolution images (figure $3 b$ and $d$ ). 
As far as spores are concerned (figure $3 \mathrm{e}, \mathrm{f}, \mathrm{g}, \mathrm{h}$ ), their surface structure is also disturbed by EP. Bacillus spores are covered by coat proteins forming parallel nanostructured named ridges (see doted lines in figure $3 \mathrm{f}$ ). These structures are clearly affected by EP (figure $3 \mathrm{~h}$ ) but no impact on roughness or cell volume were quantified (roughness was $5.5+/-2.9 \mathrm{~nm}$ for untreated spores, $4.2+/-1.7 \mathrm{~nm}$ after EP exposure and volume was $0.9+/-0.2 \mu \mathrm{m}^{3}$ for untreated cells and $0.9+/-0.3 \mu \mathrm{m}^{3}$ for exposed spores).

These two examples demonstrate the interest of AFM imaging for the fundamental elucidation of EP effect on cell morphology, shape, volume, and membrane ultrastructures.

\section{Probing biophysical modifications induced by EP}

More than an imaging tool, AFM is a force machine and when indenting the tip in the sample, nanomechanical parameters are measured. As already stated and observed on the AFM images, the mammalian cell cytoskeleton seems to be transiently destabilized by EP. The nanomechanical properties of the cells should therefore be altered too. To make this point sure, Chopinet et al. ${ }^{9}$, in 2013 measured the Young Modulus of Chinese Hamster Ovary cells before EP and monitored the cell recovery across time. Figure 4A presents 5 elasticity maps of the same CHO cell across time. EP is applied at 0 time and the same area on the cell is monitored during 35 minutes after EP. Figure 4B shows indentation force curves where the applied force is plotted as a function of the tip indentation in the cell. It clearly demonstrates the softening of the cell after EP and the recovery 35 minutes after EP. Indeed, as seen on figure 4C (histogram in the center), the mean YM drops from $18.8+/-2.0 \mathrm{kPa}$ before EP to $11.2+/-0.5 \mathrm{kPa}$ just after EP. On fixed cells, the YM of native cells is $30.0+/-2.0 \mathrm{kPa}$ and decrease to $20.5+/-1.0 \mathrm{kPa}$ after EP. The same relative difference is thus found, emphasizing 
the pitfall that absolute values of YM can constitute, and the high attention that should be paid to the conditions of YM comparisons.

During the 35 minutes of the experiment on living cells, the authors show that the mean YM increases slowly to roughly reach the initial value again. The authors explored different areas of the cells according to their position in regard to the electrodes. The hypothesis was that areas facing the positive or the negative electrodes would be differently affected. However, no differences in the YM were found on these different areas of the cell membrane, suggesting a mechanism involving more than the cell membrane. It must be noticed that the mean YM is remarquably constant if no stress is applied to the cell (left histogram on figure $4 \mathrm{C}$ ). Conversely the cell permeability induced by EP is shorter than the modification of the cells YM. The histogram on the right of figure $4 \mathrm{C}$ demonstrates that 5 minutes after EP, the cell membrane is no more permeabilized and that small molecules like propidium iodide cannot anymore go through.

A
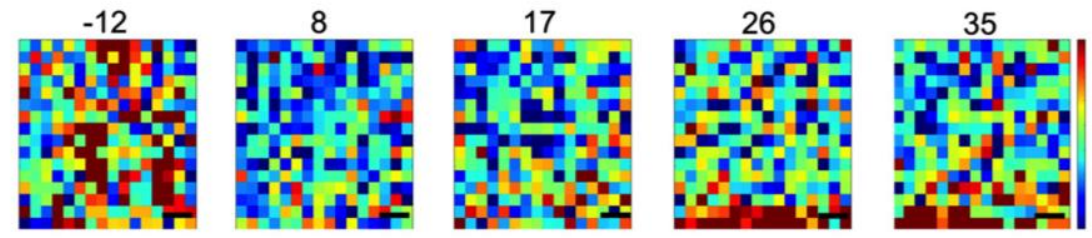

B
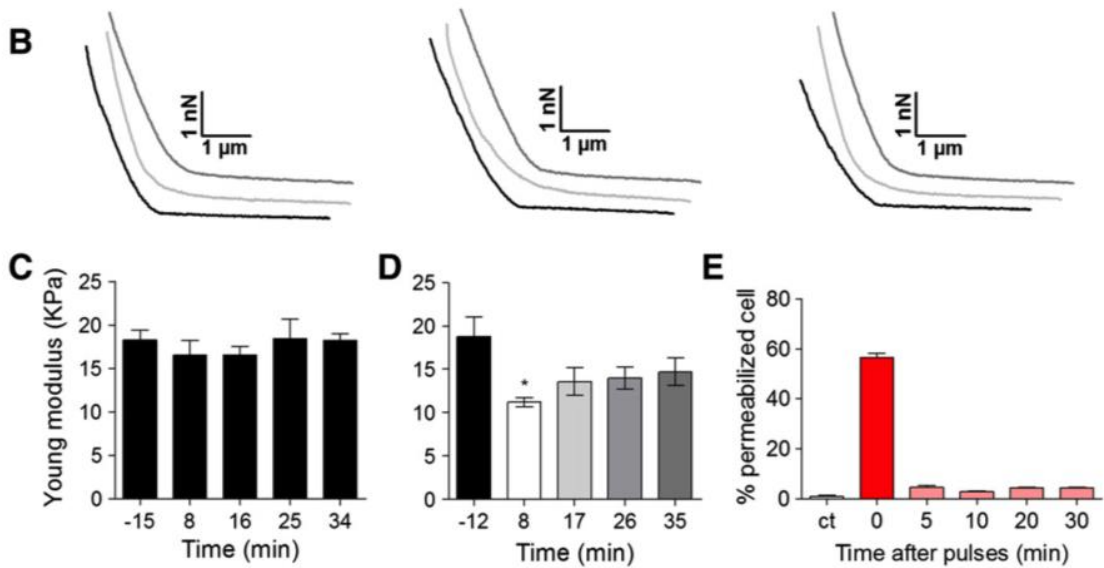

Figure 4: Probing nanomechanical properties of electroporated cells across time. EP is applied at time 0, except on $C$ where EP is not applied. A: YM maps of the same region acquired during 50 minutes (the color scale given on the right range fomr 0 to $36 \mathrm{kPa}$ ). B: typical force curves extracted from the maps presented in A. Black lines: before EP; light-grey line: 8 minutes after EP; dark-grey lines: 35 minutes after EP. C: evolution of the YM of a non puled cell across time; D: evolution of the YM of a pulsed cell 
(EP applied at time 0). unpaired t-test, *P value $=0.05 ; \mathrm{E}: \%$ of permeabilized cells across time after EP. adapted from ${ }^{9}$

Therefore, EP modifies the cell membrane but there is another mechanism responsible for the long-term modification of the cells YM. To explore the role of the actin cytoskeleton in this mechanism, the YM of latrunculin treated cells has been measured ${ }^{11}$. Indeed, latrunculin is a drug inhibiting the actin polymerization. The YM of latrunculin treated cells was found to be close to the value of cells submitted to EP: $10.6+/-0.7 \mathrm{kPa}$. Interstingly, removing the drug (latrunculin) from the medium results in an increase of the YM (figure 5D) toward values similar to cells having recovered from EP: $17.3+/-1.7 \mathrm{kPa}$. The cytoskeleton fibers are reconstructed during this process as observed on both the AFM height images and the elasticity maps respectively on figure $5 \mathrm{~A}, \mathrm{~B}$ and $\mathrm{E}, \mathrm{F}$. Combining the two treatments (latrunculin and EP) provided surprising results. When applied first, latrunculin induces a dramatic decrease in the mean YM and EP, applied in a second step, has no more impact on the cell's elasticity. On the contrary, when applied first, EP induces the usual YM decrease and lactrunculin, applied in a second step has still an impact, reducing the mean YM of the cells toward $8.3+/-0.3 \mathrm{kPa}$. This result means that EP and latrunculin both have an effect on the cytoskeleton, but not the same one! The author's interpretation is that EP destabilizes the cortical actin linked with the membrane whereas latrunclin inhibits the actin polymerization in the whole cell. This hypothesis was then reinforced by an experiment consisting in applying EP after removing latrunculin from the medium (figure 5I). In this case, cells were able to reconstruct their actin network but were unable to establish the link between the cortical actin network and the membrane, resulting in a low mean YM of $9.2+/-0.6 \mathrm{kPa}$ even 32 minutes after EP application. The AFM height image (figure 5C) and elasticity map (figure 5G) confirm that, even if the actin polymerization occurs, no cytoskeleton fibers can be observed. 

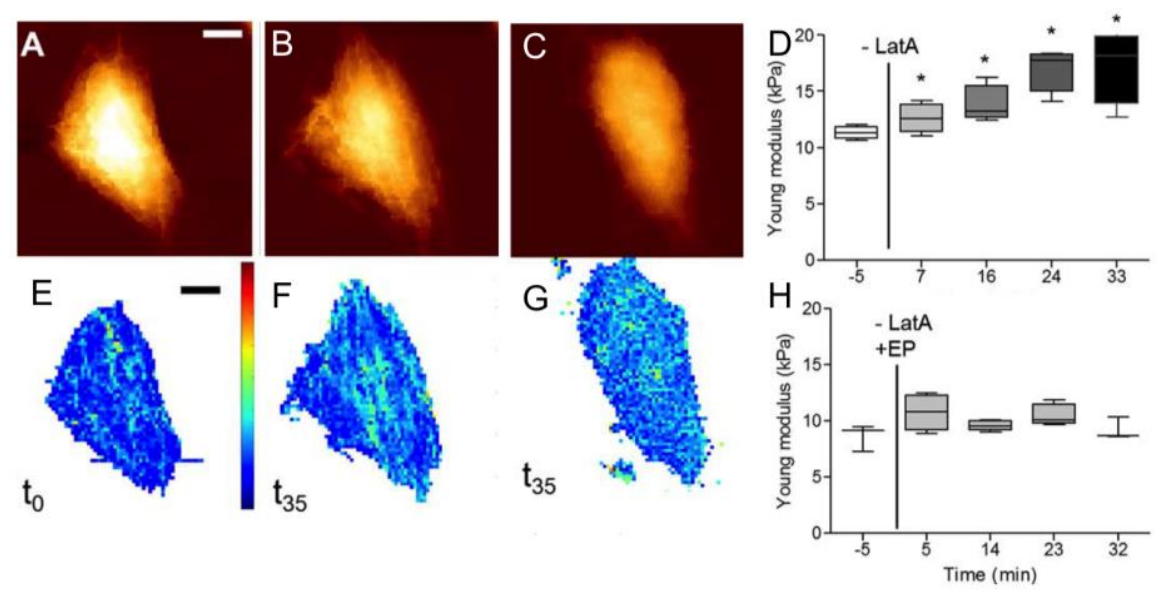

Figure 5: Topography and elasticity of cells exposed to latrunculin and EP. A, B and C are AFM QI height images of a latrunculin treated cell (A), the same cell 35 minutes after latrunculin removal $(B)$ and a cell 35 minutes after latrunculin removal but electroporated at time 0 . E, $F$ and $G$ are the respective elasticity maps of the precedent conditions. The color scale given on the right of $E$ range from 0 to $60 \mathrm{kPa}$. D: Evolution of the YM after latrunculin removal. Unpaired $t$ test relative to the point before $t=0$ : * $P$ value $<0.05$; H: Evolution of the YM after luntrunculin removal if $\mathrm{EP}$ is applied at time 0 . Adapted from ${ }^{11}$

In 2014 Thompson et al. ${ }^{13}$, used a similar strategy to explore the effect of nanosecond Pulsed Electric Fields (nsPEFs) (50 to 100 pulses of $10 \mathrm{~ns} ; 150 \mathrm{kV} / \mathrm{cm}$ ) on the actin cortex of cells. In this work $\mathrm{CHO}-\mathrm{K} 1$ cells elasticity is measured using a colloidal probe before and after EP, on native or latrunculin treated cells. Colloidal probes are AFM cantilevers terminated by a spherical bead (here made of borosilicate, and of $5 \mu \mathrm{m}$ in diameter). The contact area between the probe and the sample is thus much higher than with pyramidal probes what is an advantage to measure global modifications, but is a drawback when it comes to measure fine structures like cytoskeleton fibers. Despite the different applied pulses and probe, they found quite similar results: nsPEFs induce a decrease in the cell elastic modulus of $50 \%$. And Latrunculin A has the same qualitative effect but is quantitatively higher. The cell elasticity of latrunculin treated cells is, indeed, $75 \%$ lower than for healthy cells. Interestingly, the application of nsPEFs on latrunculin treated cells had no effect on the cells elasticity. These results are in line with other reports already described here and confirm that EP has an action on the cells mechanical properties that is most probably mediated by modification at the cytoskeleton level. 
In the case of microorganisms, the biophysical properties of the interface are driven by the cell wall ${ }^{8}$. To continue with Bacillus pumilus, which is a Gram-positive bacterium, the cell wall of the vegetative form is made of a thick $(50 \mathrm{~nm})$ layer of peptidoglycan. The peptidoglycan has been described to be coiled and super coiled around the cells ${ }^{25}$. The stiffness map presented in figure 6 a adapted from ${ }^{12}$ confirms this nanoscale organization of the peptidoglycan (doted lines correspond to peptidoglycan cables). After EP (figure 6d), the stiffness map does not show any peptidoglycan organization anymore. The mean stiffness did not really changed (increasing from $0.08 \mathrm{~N} / \mathrm{m}$ before EP to $0.09 \mathrm{~N} / \mathrm{m}$ after EP) but the surface became heterogeneous as evidenced by the standard deviation increasing from $0.01 \mathrm{~N} / \mathrm{m}$ before EP to 0.03 after EP. In this work, the authors also studied the cell surface hydrophobicity as probed by chemical force microscopy $(\mathrm{CFM})^{20}$ (figure $6 \mathrm{~b}$ and h). They measured a mean adhesion force of $2.1+/-1.2 \mathrm{nN}$ (figure 6b) on untreated vegetative bacteria, $0.2+/-0.3 \mathrm{nN}$ on untreated spores (figure $6 \mathrm{f}$ ), $0.2+/-0.2 \mathrm{nN}$ on pulsed vegetative cells (figure 6e) and $0.1+/-0.1 \mathrm{nN}$ on pulsed spores (figure 6f). The CFM technology clearly demonstrates in this study the impact of EP on both vegetative forms and spores of Bacillus pumilus hydrophobicity, which is a key parameter in adhesion and biofilm formation.
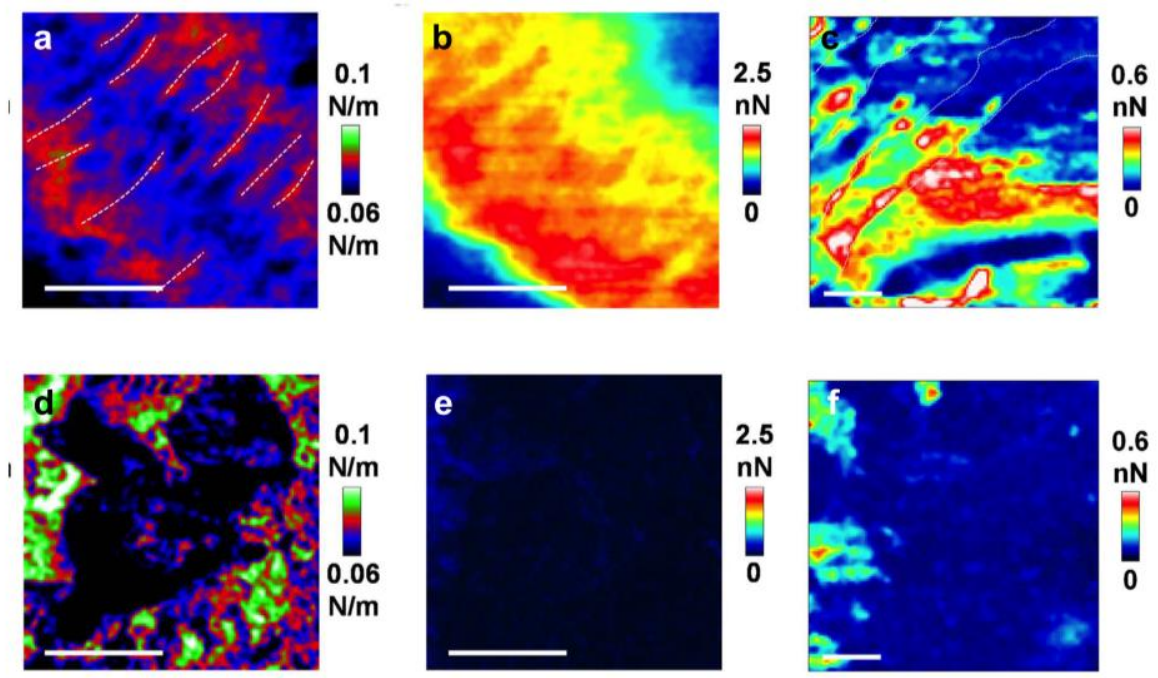
Figure 6: AFM stiffness (a, d) and adhesion (b, c, e and f) maps recorded on Bacillus pumilus vegetative cells $(a, b, d$ and $e)$ and spores $(e, f)$. The white bar represents $200 \mathrm{~nm}$ in $a, b, d, e$ and $100 \mathrm{~nm}$ in $\mathrm{c}$ and f; the color scale next to each image corresponds to the stiffness $(a, d)$ or the adhesion force $(b, c, e$ and $f)$ adapted from ${ }^{12}$

\section{Concluding remarks}

Very few studies devoted to EP are using AFM. Works presented here nevertheless demonstrate the interest of this quite new technology in the field. Indeed, AFM has proven its ability to image the modifications induced by EP both on mammalian and bacteria cells. Moreover it gives access to nanomechanical information, with a spatial resolution, that is crucial to better understand the fundamental mechanisms of EP. On bacteria the measure and mapping of adhesions, in this case of hydrophobicity, could have important applied consequences, eg in the food industry.

In the future it would be useful to develop a device to apply electric pulses on the AFM setup. Then combining AFM and high resolution optical imaging it would be interesting to analyze the permeabilization of cells membranes toward anti-cancerous drugs or nucleic acids. 


\section{REFERENCES}

1. Wong, T.-K. \& Neumann, E. Electric field mediated gene transfer. Biochem. Biophys. Res. Commun. 107, 584-587 (1982).

2. Testori, A., Rossi, C. R. \& Tosti, G. Utility of electrochemotherapy in melanoma treatment. Curr. Opin. Oncol. 24, (2012).

3. Bodles-Brakhop, A. M., Heller, R. \& Draghia-Akli, R. Electroporation for the Delivery of DNA-based Vaccines and Immunotherapeutics: Current Clinical Developments. Mol. Ther. 17, 585-592 (2009).

4. Teissie, J., Golzio, M. \& Rols, M. P. Mechanisms of cell membrane electropermeabilization: A minireview of our present (lack of ?) knowledge. Biochim. Biophys. Acta BBA - Gen. Subj. 1724, 270-280 (2005).

5. Binnig, G. \& Quate, C. F. Atomic force microscope. Phys Rev Lett. 56, 930-933 (1986). 6. Hansma, P. K., Elings, V. B., Marti, O. \& Bracker, C. E. Scanning tunneling microscopoy and atomic force microscopy : application to biology and technology. Science 247, 209-216 (1988).

7. Gerber, C. \& Lang, H. P. How the doors to the nanoworld were opened. Nat. Nanotechnol. 1, 3-5 (2006).

8. Pillet, F., Chopinet, L., Formosa, C. \& Dague, É. Atomic Force Microscopy and pharmacology: From microbiology to cancerology. Biochim. Biophys. Acta BBA - Gen. Subj. 1840, 1028-1050 (2014).

9. Chopinet, L., Roduit, C., Rols, M.-P. \& Dague, E. Destabilization induced by electropermeabilization analyzed by atomic force microscopy. Biochim. Biophys. Acta BBA Biomembr. 1828, 2223-2229 (2013).

10. Chopinet, L., Formosa, C., Rols, M. P., Duval, R. E. \& Dague, E. Imaging living cells surface and quantifying its properties at high resolution using AFM in $\mathrm{QI}^{\mathrm{TM}}$ mode. Micron 48, 26-33 (2013).

11. Louise, C., Marie-Pierre, R. \& Etienne, D. AFM sensing cortical actin cytoskeleton destabilization during plasma membrane electropermeabilization. Cytoskeleton n/a-n/a (2014). doi:10.1002/cm.21194

12. Pillet, F., Formosa-Dague, C., Baaziz, H., Dague, E. \& Rols, M.-P. Cell wall as a target for bacteria inactivation by pulsed electric fields. Sci. Rep. 6, 19778 (2016).

13. Thompson, G. L., Roth, C., Tolstykh, G., Kuipers, M. \& Ibey, B. L. Disruption of the actin cortex contributes to susceptibility of mammalian cells to nanosecond pulsed electric fields. Bioelectromagnetics 35, 262-272 (2014).

14. Neuman, K. C. \& Nagy, A. Single-molecule force spectroscopy: optical tweezers, magnetic tweezers and atomic force microscopy. Nat. Methods 5, 491-505 (2008).

15. El Kirat, K., Burton, I., Dupres, V. \& Dufrene, Y. F. Sample preparation procedures for biological atomic force microscopy. J. Microsc. 218, 199-207 (2005).

16. Touhami, A., Nysten, B. \& Dufrêne, Y. F. Nanoscale Mapping of the Elasticity of Microbial Cells by Atomic Force Microscopy. Langmuir 19, 4539-4543 (2003).

17. Dague, E. et al. Assembly of live micro-organisms on microstructured PDMS stamps by convective/capillary deposition for AFM bio-experiments. Nanotechnology 22, 395102 (2011).

18. Formosa, C. et al. Generation of living cell arrays for atomic force microscopy studies. 
Nat. Protoc. 10, 199-204 (2015).

19. Ahimou, F., Denis, F. A., Touhami, A. \& Dufrêne, Y. F. Probing microbial cell surface charges by atomic force microscopy. Langmuir 18, 9937-9941 (2002).

20. Dague, E. et al. Chemical force microscopy of single live cells. Nano Lett 7, 3026-3030 (2007).

21. Hinterdorfer, P., Baumgartner, W., Gruber, H. J., Schilcher, K. \& Schindler, H. Detection and localization of individual antibody-antigen recognition events by atomic force microscopy. Proc. Natl. Acad. Sci. 93, 3477-3481 (1996).

22. Hinterdorfer, P. \& Dufrêne, Y. F. Detection and localization of single molecular recognition events using atomic force microscopy. Nat. Methods 3, 347-355 (2006).

23. Dufrêne, Y. F., Martínez-Martín, D., Medalsy, I., Alsteens, D. \& Müller, D. J.

Multiparametric imaging of biological systems by force-distance curve-based AFM. Nat. Methods 10, 847-854 (2013).

24. Hayhurst, E. J., Kailas, L., Hobbs, J. K. \& Foster, S. J. Cell wall peptidoglycan architecture in Bacillus subtilis. Proc. Natl. Acad. Sci. 105, 14603-14608 (2008). 